\title{
THE ILEO-CAECAL MASS
}

J. B. M. RoBeRTS, M.B., F.R.C.S.

Surgical Registrar, the Royal Infirmary, Sheffield

For the final elucidation of many problems of intra-abdominal diagnosis the surgeon is often dependent upon laparotomy. The vague shadows of the X-ray screen are then replaced by the realities of a diseased organ and the ephemeral boundaries of a palpated mass by its precise pattern. Yet there are cases when even this scrutiny fails to reveal the nature of the disease, and in few places is this more true than when there is exposed a mass, in the region of the ileocaecal junction. Here many lesions may produce an identical picture, and it is upon the shoulders of the histologist that the role of diagnostician must fall.

It was therefore decided that an analysis of the causes of a series of such masses might be of general interest, and this is now presented. The material used represents 85 consecutive patients in whom a mass in the ileo-caecal region was found at operation, between the years 1949 and 1958 at the Royal Infirmary, Sheffield. For the sake of brevity the obvious appendix abscess has been excluded, and only those which were a source of mistake or doubt have been included.

A superficial examination of the diagnoses in this series reveals three main classes of pathology: carcinoma of the caecum, regional ileitis, and a miscellaneous group which will be discussed in more detail below.

The frequency with which cases occurred within these groups is depicted in Table $\mathrm{r}$.

Apart from appendix abscess, carcinoma of caecum was the commonest source of an ileocaecal mass. It was, however, present less frequently than was suspected at laparotomy by the surgeon, being mimicked closely by several lesions in the caecum of a simple inflammatory nature. The diversity of these caecal pathologies is illustrated by examples of lesions which were resected as carcinomata.

Table I.-Analysis of diagnoses

Carcinoma of caecum .. $\quad$. . $\quad \ldots \quad 46(54.11 \%)$

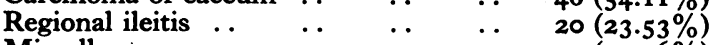
Miscellaneous group $\quad \ldots \quad \ldots . \quad \ldots \quad 19(22.36 \%)$

Total number of cases $\quad \ldots \quad \ldots \quad \overline{85(100 \%)}$

\section{Lesions Resected as Carcinomata}

(I) Solitary diverticulum of the caecum (two cases).

(2) Crohn's disease of the caecum (two cases).

(3) Submucous lipoma of the caecum with overlying ulceration (two cases).

(4) Benign adenoma of the caecum.

(5) Tuberculous ulcer of the caecum.

(6) Argentaffinoma of the caecum.

(7) Fruit stone impacted in the base of the caecum.

(8) Appendix granuloma.

Though at operation it was often possible to make the correct diagnosis, as where typical changes of acute regional ileitis, ceasing abruptly at the ileo-caecal valve, were found, there re-o mained cases where accurate diagnosis, even in retrospect, appeared impossible and mistakes were made. The three groups mentioned above have therefore been analysed under various headings in the hope of determining some factors which might facilitate diagnosis.

The miscellaneous group of cases, it may be argued, cannot be considered together for the purpose of comparison. This method is used, however, mostly as an initial superficial examination to determine if any gross difference exists between cases of carcinoma and the rest.

\section{Age Incidence}

The most obvious factor for initial consideration appears to be the age incidence. Cases of carcinoma ranged from the early thirties to 90 , with a peak incidence in the 50 to 70 -year-old group. The range of the cases of regional ileitis was from 17 to 60 -here the peak incidence occurred about the age of 30 . The tendency for regional ileitis to occur in a younger age group than does carcinoma is well known, yet there is obviously an overlap of the two diagnoses, especially in the patients aged between 30 and 60 . The miscellaneous group further confused the picture by showing two peaks of common incidence which closely coincided with the peaks of the other two groups. 


\section{Sex Incidence}

The slightly higher incidence of regional ileitis in the male, mentioned by Crohn (1949), is confirmed in this small series. Crohn recorded 56 per cent. of his cases in male patients and 12 (6o per cent.) of the 20 cases presented here were in men. Both carcinoma of the caecum and the miscellaneous group of pathologies were more common amongst women. 58.7 per cent. of the cancer cases and 57.9 per cent. of the miscellaneous series being women. In a review of cases of large bowel carcinoma Smiddy and Goligher (1957) found 53 (52.47 per cent.) of ror cases of carcinoma of the caecum to be women.

This does not confirm any definite tendency to sex variation in the incidence of this condition.

\section{Incidence and Duration of Symptoms}

Pain was the dominant symptom, either alone or in association with some change in bowel habit, in 68 ( 80 per cent.) of the 85 cases. In all three groups a central abdominal pain, most commonly periumbilical or epigastric in site, occurred twice as frequently as did pain in the right side of the abdomen. It is noticeable that in approximately half of each group the presenting symptom was pain alone. This was not associated with any spontaneously volunteered history of change in bowel habit, though in some cases such a change was elicited in response to specific questioning. A change in bowel habit without some pain was uncommon, occurring in only 6 ( 13 per cent.) of the cases of carcinoma and 2 (ro per cent.) of the ileitis group. It was not found in the miscellaneous group.

More detailed consideration of the change of bowel habit revealed that diarrhoea was by far the commonest form it took. It was five times as common as constipation in the cancer cases, and constipation was not seen in any of the cases of Crohn's disease. Diarrhoea occurred in half of the miscellaneous series, and constipation in a quarter. It is difficult to find much significance in the presence of constipation, as this is so frequently mentioned by patients who have no alimentary pathology, and the diarrhoea, though significant, is common to all groups.

The duration of symptoms was very variable, being in all groups from several hours to three years or more. Carcinoma of caecum appeared to follow two definite patterns. Twenty-four (52.17 per cent.) of the cases presented with a history of less than three months, whilst i 8 (39.13 per cent.) had a history lasting from one to three years. The history in Crohn's disease was for six to 12 months in half of the cases, though some few cases presented in an acute form, and others remembered intermittent pain and diarrhoea last- ing for several years. Six ( $31.5^{8}$ per cent.) of the miscellaneous group presented with brief histories of hours or a few days, but the rest were as variable as the other groups.

Melaena occurred once in each group of cases, but was on no occasion of sufficient severity to merit transfusion.

Consideration of the incidence of the major presenting symptoms reveals no definite way to distinguish between the conditions on the preoperative history, though it was found that none of the miscellaneous group presented with general symptoms of toxaemia, malaise, and anaemia, whilst this did occur occasionally amongst the cases of carcinoma.

\section{Incidence of Pre-operative Mass (Table 2)}

Pre-operative palpation of the abdomen demonstrates that within the limits of this small series the finding of a mass in the right iliac fossa is more in favour of carcinoma than of a lesion from the miscellaneous group. Only seven (I 5.2 per cent.) of the 46 cases of carcinoma did not present a palpable mass in the unanaesthetized patient, whilst 12 (63.I per cent.) of the 19 in the miscellaneous group had no such mass. This then provides the most definite lead so far, but it is not an absolute guide.

Table II.-Incidence of Pre-operative Mass

\begin{tabular}{|c|c|c|}
\hline & Mass & No Mass \\
\hline $\begin{array}{l}\text { Carcinoma of caecum.. } \\
\text { Regional ileitis } \\
\text { Miscellaneous .. }\end{array}$ & $\begin{array}{r}39(84.34 \%) \\
12(60.00 \%) \\
7(36.84 \%)\end{array}$ & $\begin{array}{r}7(15.66 \%) \\
8(40.00 \%) \\
12(63.16 \%)\end{array}$ \\
\hline
\end{tabular}

\section{Discussion}

The mass in the ileo-caecal region is a common source of diagnostic error. This is chiefly because a wide range of pathologies - may all produce a sufficient mass of inflammatory adhesions and thickening to give an identical picture. Even when the lesion remains discrete and there is minimal surrounding reaction, caecal pathologies must frequently be mistaken for carcinomata. The adenoma or lipoma, with some overlying ulceration, felt through the caecal wall, may easily be taken to represent early malignant change.

In an attempt to clarify the picture, various factors, including sex incidence, age incidence and symptomatology, have been considered, but none is of definite diagnostic help.

$\mathrm{X}$-ray examination is often useful, but it may also be misleading. In one case of the series a carcinoma of the caecum was present in association with two diverticulae of the caecum. Thus the radiological demonstration of caecal diverticulae cannot be taken to mean that a caecal 
mass is due to diverticulitis. Barium enema undertaken in another case because of intermittent subacute intestinal obstruction associated with a mass in the right iliac fossa revealed a filling defect in the caecum, thought to be due to a carcinoma. At operation changes typical of regional ileitis were found. Right hemicolectomy was performed, and careful examination of the specimen revealed only superficial mucosal ulceration in the caecum.

The presence of secondary deposits in the liver or in the peritoneal cavity strongly suggests that the mass is the primary growth. Discrete nodules in the liver are not, however, synonymous with neoplastic deposits, nor does it follow absolutely that there is not a primary source elsewhere because a mass is felt in the ileo-caecal region. In one instance such a nodule was found at laparotomy and was thought to confirm the diagnosis of carcinoma-later microscopy of the removed hemicolectomy specimen revealed the changes to be inflammatory, and two years later the patient showed no clinically detectable signs of malignancy.

Involvement of the regional lymph nodes may be either inflammatory or neoplastic in origin, and it is impossible to distinguish between the two with certainty on inspection at operation. Firmly enlarged glands were present in most of the non-malignant cases, whilst in cases of carcinoma of the caecum the glandular enlargement was frequently inflammatory and not neoplastic. Glandular involvement is then of little help in establishing a diagnosis.

Within this series interesting diagnostic problems were posed in three areas of the region under consideration:

(I) In the body of the caecum-especially on its posterior wall.

(2) At the orifice of the appendix.

(3) In the terminal inches of the ileum.

\section{(x) In the Body of the Caecum}

Carcinoma of the caecum commonly presents in the following forms: The proliferative papilliferous lesion; the malignant ulcer; as a diffuse mass adherent to surrounding structures.

All three forms were closely paralleled by benign lesions. The papilliferous lesion by an adenoma of the caecum, and twice by submucous lipomata with overlying mucosal ulceration.

In each case resection of the right colon was undertaken for what was believed to be a carcinoma.

The malignant ulcer by a large tuberculous ulcer on the posterior wall of the caecum, and finally the diffuse malignant mass was mimicked by several conditions including diverticulitis, hypertrophic ileo-caecal tuberculosis, Crohn's disease of the caecum, and appendix granuloma.

Detailed consideration of some of the lesions reveals several points which are helpful in differentiating them from carcinoma.

(a) Solitary Diverticulitis of the Caecum.Whilst this is not a common condition, it is probable that it occurs more frequently than many suspect. References to it in recent literature are increasing (Case and Shea, 1953; Waite, 1954). All authors are in agreement that whilst many

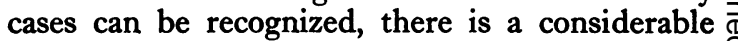
number which cannot be differentiated from carcinoma except perhaps by the use of a frozen section technique.

Frequently the diverticulum is sited on the antero-lateral aspect of the caecum (Reid, 195I), and when early inflammatory changes only have $\bar{C}$ occurred its recognition is simple. Reid states, 3 however, that the other common site for the diverticulum is on the posterior surface of the caecum. Here, with much surrounding induration, differentiation from carcinoma may be impossible. Moon and Schenk (1945) are quoted by $\infty$ Reid as stating that palpation of the orifice of the diverticulum makes diagnosis certain. He points out, however, that whilst this is true in some cases, such palpation may well prove impossibfe in the grossly indurated case.

The pre-operative picture gained from the literature is one closely simulating appendicitß in 85 per cent. Whilst this does occur with of carcinoma of the caecum, it is far less commom. Bennett-Jones (1937) confirms these views, and whilst stressing the importance of the condition as it so frequently mimics the much commoner appendicitis, also recognizes that it may present as a mass indistinguishable from carcinoma. Unger (1953), reviewing 132 cases, stresses that distinction from cancer may be impossible, and 26 (19.7 per cent.) of his series were resected as malignant lesions. Grace's (1938) case of diverticulitis on the posterior caecal wall was also resected as carcinomatous.

In discussing the treatment of this condition, Waite (1954) states that simple excision or inversion of the diverticulum, if recognized, is sufficient. He quotes a mortality rate of about i I per cent. for the operation of right hemicolectomy as the strongest argument against radical surgery if not absolutely necessary.

To underline finally the difficulty that may be experienced in making an accurate diagnosis, one finds that Willis (1948), with his vast experience, $N$ states that even at autopsy he has been mistaken ${ }_{\omega}^{N}$ in the differentiation between diverticulitis and cancer until microscopical examination of tissue 0 removed had been undertaken.

(b) Submucous Lipoma.-Aird (1957) states that $\stackrel{\oplus}{?}$ 
these are usually pedunculated, but this was not so in either of the two cases seen. Both had overlying mucosal ulceration and infection. Being sited on the posterior caecal wall it is not surprising that both were mistaken for carcinoma and treated by hemicolectomy.

(c) Crohn's Disease.-This can usually be distinguished easily from carcinoma, but in cases where the main impact is on the caecum, and only the immediately adjacent ileum is involved, the whole inflammatory mass may be mistaken for cancer. Armitage and Wilson (1950) mention this in their review of the condition as do many other authors.

(d) Actinomycosis.-No example of ileo-caecal actinomycosis presented during the ro years under survey. This is perhaps surprising, though Armitage and Smith (1954) point out that it is not a common condition. They could find only nine proven cases of abdominal actinomycosis in 15 years from a population of 500,000 to $75^{\circ}, 000$. Zachary Cope (1956), with his unique experience of the condition, states that all early cases of actinomycosis have been resected as cancer, and that it is only in the late case with multiple abscess and sinus formation that it is likely to be recognized clinically.

(e) Hypertrophic Ileo-caecal Tuberculosis.-In discussing the differential diagnosis from carcinoma Aird (1957) mentions the radiological changes. In hyperplastic tuberculosis the filling defect at barium enema examination tends to be long and tapering at both ends, whilst in cancer it is shorter and more abrupt. Calcified mesenteric glands may be found with any of the pathologies in this region as the end-result of a long past attack of tuberculous mesenteric adenitis. They have no diagnostic significance. Randall and Spalding (1946) have described co-existing tuberculosis and carcinoma of the colon.

(f) Appendicular Granuloma.-Le Brun (1958) presented seven such cases, six of which were diagnosed as carcinoma at operation. The distinction between the two conditions is difficult.

(g) Simple Ulcer of the Caecum was not seen in this series. Cameron (1939) recorded 23 cases he had gathered from the literature and found that they occurred most commonly opposite the ileo-caecal valve, or on the anterior caecal wall at the level of the valve; 65 per cent. presented following perforation. Most were sufficiently discrete to be recognized as benign, and were treated by local resection. Case and Shea (1953) believe that many of these ulcers may be the end-resuilt of an attack of inflammation in a solitary caecal diverticulum.

\section{(2) At the Orifice of the Appendix}

The presence at laparotomy of a distended appendix in association with an inflamed mass in the immediately adjacent caecum raises a further diagnostic problem. This picture occurred six times. On four occasions the obstruction was malignant, in one it was due to appendicular calculi about the orifice of the appendix, and in the last instance was due to the impaction of a fruit stone in the orifice. Two of these case histories illustrate the complexity of the problem.

A previously healthy man, aged 30 , was admitted with a history of 24 hours' central, colicky abdominal pain associated with vomiting. Shortly prior to admission the pain had moved to the right iliac fossa. Examination revealed tenderness and guarding in the right iliac fossa, and a preoperative diagnosis of acute appendicitis was made. At operation an obstructive appendicitis was found, and associated with this was an extremely hard mass present in an indurated caecum which showed adhesions to the lateral wall of the peritoneal cavity. This was thought to represent a carcinoma of the caecum which had obstructed the orifice of the appendix. Right hemicolectomy was therefore performed, and the patient unfortunately died following a prolonged paralytic ileus. Inspection of the mass on removal of the specimen revealed it to contain a fruit stone about the size of that of an apricot which had obstructed the appendicular orifice, and had produced the caecal induration.

To stress the difficulty further, another case from the series is offered for comparison. A previously healthy man of 46 was admitted following central abdominal colic associated with anorexia and nausea which had been present for four days. There had been slight diarrhoea for the two days before admission. Examination revealed tenderness in the right iliac fossa where there was also muscle guarding which could be overcome to make apparent a very tender but mobile mass. At operation an obstructive appendicitis was found and the caecum at the base of the appendix was hard and indurated. Local resection of the appendix with the adjacent caecum was performed. Histology of the removed specimen demonstrated the presence of a carcinoma of the caecum. A formal right hemicolectomy was therefore undertaken, and histology of this specimen failed to demonstrate any malignant tissue, yet this patient was dead from carcinomatosis four years later. Without opening the caecum it would appear to have been impossible to distinguish between these two cases. As the first patient was aged only 30 , it is possible that a more conservative attitude might have been adotped, yet the surgeon was sure of his diag- 
nosis, and carcinoma is not rare even at this relatively young age.

\section{(3) In the Terminal Ileum}

Whilst the commonest source of a mass in the ileum adjacent to the ileo-caecal valve is matted coils of small intestine involved by regional ileitis, various other pathologies do occur. There were 20 cases of regional ileitis in this series, but there were also five other pathologies which presented the same picture:

(i) Carcinoma of the ileum.

(ii) Argentaffinoma of the ileum.

(iii) Foreign body reaction to silver paper impacted in a diverticulum of the ileum with perforation.

(iv) Non-specific granuloma of the ileum-20 years after radium treatment of the uterus.

(v) A discrete secondary deposit at the ileocaecal angle from a previously treated carcinoma of the body of the uterus.

All were resected as carcinomata except the first case, which was initially treated by ileotransverse colostomy in the belief that it was a tuberculous stricture. When re-explored a large mass was present which proved to be a primary carcinoma of the ileum.

Seventeen distinct pathologies were met with in these 85 cases, and there are various other conditions such as actinomycosis, endometrioma, and amoeboma, which might have added to this number. It is then not surprising that the most thorough surgeon can be mistaken in dealing with the pathology of this region. In view of this at times impossible diagnostic task, it is fortunate that many of the conditions considered are well treated by right hemicolectomy. Awareness of the diversity of conditions which occur here may occasionally prevent unnecessarily radical surgery, it will certainly make this junction point of the alimentary tract one of intrigue to the clinician.

\section{Summary}

Eighty-five cases which presented with a mass in the ileo-caecal region are discussed, demonstrating the difficulties in diagnosis. The complexity of the subject is made clear in a review of the relevant literature. It is concluded that, owing to the similarity of the clinical pictures produced by various different pathologies, some diagnostic errors are inevitable.

\section{Acknowledgments}

It is a pleasure to acknowledge my debt to the consultant surgical staff of the Royal Infirmary, Sheffield, who permitted me to study their cases, and especially to Mr. Clifford Jones for his continued advice and encouragement.

\section{BIBLIOGRAPHY}

AIRD, I. (r957), 'Companion in Surgical Studies,' and edition, Edinburgh and London, Livingstone.

ARMITAGE, G., and WILSON, M. (1950), Brit. F. Surg., 38, 189. ARMITAGE, G., and SMITH, I. (r954), Ibid., 42, 77.

BENNETT-JONES, M. J. (1937), Ibid., 25, 60.

CAMERON, J. R. (1939), Ibid., 26, 527.

CASE, T. C., and SHEA, E. C. (1953), Amer. F. Surg,, 85, 134. COPE, Z. (1956), in ' Textbook of British Surgery,' Vol. I, London, Heinemann.

CROHN, B. B. (1949), ' Regional Ileitis,' London, Staples Press. GRACE, A. J. (1938), Brit. ₹. Surg., 26, 200.

LE BRUN, H. I. (1958), Ibid., 46, 37.

RANDALL, K. J., and SPALDING, J. E. (1946), Ibid., 33, 372( REID, D. R. K. (195I), Ibid., 39, 76.

SMIDDY, F. G., and GOLIGHER, J. C. (1957), Brit. Med. Э., I, 793 .

UNGER, H. M. (1953), Amer. F. Surg., 85, 781.

WAITE, V. C. (1954), Ibid., 88, 721.

WILLIS, R. A., (1948), 'Pathology of Tumours,' London, Butterworth. 